\title{
Cerebellum and Endocannabinoid Receptors: A New Possible Neurobiological Link For Mitragynine (Mitragyna Speciosa Korth) Abuse Liability
}

\author{
Jayabalan Nanthini ${ }^{1,2}$, Nurul Iman W. Ismail ${ }^{1,2}$, Sharif M. Mansur ${ }^{3}$, Christian P. Müller ${ }^{4}$, Mustapha \\ Muzaimi ${ }^{12^{*}}$
}

${ }^{1}$ Department of Neurosciences, School of Medical Sciences, Universiti Sains Malaysia

${ }^{2}$ Centre for Neurosciences Service and Research, Universiti Sains Malaysia

${ }^{3}$ Centre for Drug Research, Universiti Sains Malaysia

${ }^{4}$ Department of Psychiatry and Psychotherapy, Friedrich-Alexander-University Erlangen-Nuremberg, Germany

*Corresponding Author: Muzaimi Mustapha, Department of Neurosciences, School of Medical Sciences, Universiti Sains Malaysia, 16150 Kubang Kerian Kelantan, Malaysia. Tel: +6097676309; Fax:+6097673833; E-mail: mmuzaimi@usm.my

\begin{abstract}
Drug abuse is a major concern worldwide and has remained so for decades. Beside synthetic drugs, the misuse of psychoactive compounds from natural products as drugs of abuse contributes to this incessant concern. Reports on misuse of mitragynine, an alkaloid from the plant Mitragyna speciosa Korth (Kratom or Ketum) as a substituent for opioid withdrawal syndrome are becoming prevalent in recent years, and extended beyond the geographical boundaries of the plant. Although there are comprehensive data available on chemical and pharmacological properties of mitragynine particularly on its opioid-like analgesic property, the mechanism of actions for its abuse liabilities remain unclear. This article presents the plausible leads for mitragynine abuse liability by reviewing the established roles of endocannabinoid system as a molecular target where the opioids and cannabinoids act. These leads build for the arguments to posit the involvement of ubiquitous endocannabinoid receptors within the cerebellum and the evidences for cerebellar non-motor functions (brain reward processes, drug-induced long term memory and plasticity, and structural alterations linked to addiction) towards a potential new player as the basis for abuse liability of Kratom, accumulating to addiction.
\end{abstract}

Received Date: August 12, 2015

Accepted Date: October 18, 2015

Published Date: October 20, 2015

Citation: Nanthini, J., et al Cerebellum and Endocannabinoid Receptors: A New Possible Neurobiological Link for Mitragynine (Mitragyna Speciosa Korth) Abuse Liability. (2015) J Addict Depend 1(1): 15- 21.

DOI: $10.15436 / 2471-061 X-15-004$

Keywords: Cerebellum; Endocannabinoid; CB1 Receptor; Mitragynaspeciosa; Mitragynine; Addictive Behaviour

\section{Introduction}

Global misuse of psychoactive phytochemical mitragynine from Mitragyna speciosa is an emerging trend. Mitragyna speciosa is a rubiaceous herbal tree indigenous to swamp areas in the Southeast Asia, notably Malaysia and Thailand ${ }^{[1,2]}$. Known to the locals as "Ketum or Biak-Biak" among the Malaysians, or "Kratom" 'Kakuam', 'Ithang' or 'Thom' in Thailand ${ }^{[3]}$, the plant has been traditionally used as a regional folk remedy. First described and named by Dutch botanist Pieter Willem Korthals, this plant has clustered flowers that are yellow and each of the flower encompassed approximately 120 florets $^{[2]}$. The active part of Ketum is their dark green leaves which are oval in shape with a slender end (ovate-acuminate) and are able to grow over 7 inches in long and 4 inches wide ${ }^{[2]}$. Since time immemorial, the leaves of this plant have been used for ailments such as fever, cough, pain and diarrhoea, to deworm, malaria, to improve blood circulation and also even to treat diabetes and hypertension ${ }^{[4,5]}$. Besides, Ketum is also popular among the open-field labourers who use Ketum for heightened work endurance under the hot sun ${ }^{[2]}$. In addition, the use of Ketum as a potential psychoactive dietary supplement is evident from anecdotal reports as early as the 19th century ${ }^{[2]}$. In recent times Ketum is being utilised for opium substitute and weaning addicts off morphine and has since liable to addictive and misuse potentials.

Copy rights: (C2015 Nanthini, J. This is an Open access article distributed under the terms of Creative Commons Attribution 4.0 International License. 
In this paper, we elaborate on two potent alkaloids of Ketum and their known opioid-like effects. We recapitulate the evidences and findings on reciprocal interaction between opioids and endocannabinoids, and posit the possible neurobiological link in exerting the addictive potential of mitragynine. Building from this basis, we extend the current understanding on the role of cerebellum in higher cognitive functions through the involvement of abundant cerebellar endocannabinoid CB1R, particularly in regulating the reward and drug-seeking behaviour. The objective of this review, therefore, is to propose plausible leads involving cerebellar endocannabinoid CB1R and mitragynine, as the potential new basis for mitragynine/Ketum abuse liabilities.

\section{The Active Constituents of Mitragynaspeciosa}

About 40 compounds have been extracted from the leaves of Ketum and the mitragynine is the most active, primary constituent of this plant ${ }^{[6]}$. Apart from mitragynine, other alkaloids of Ketum previously reported include speciogynine, speciociliatine, paynantheine and 7-hydroxymitragynine $\mathrm{e}^{[7]}$. The 7-hydroxymitragynine is a more potent but less abundant congener of mitragynine ${ }^{[2,7]}$. It is also recognised that the presence of different types of alkaloids and their percentage varies based on their geographical region ${ }^{[7,8]}$. For instance, the total amount of mitragynine derived from Ketum of Thai origin accounts for about $66 \%$ while about $12 \%$ of mitragynine from Ketum of Malaysia origin ${ }^{[7]}$. The chemical structure of mitragynine had been fully determined by Zacharias using the X-ray crystallography in $1965^{[9]}$. The identification of lowest energy conformation through computational study further validated the X-ray crystal structure geometry of this alkaloid ${ }^{[10]}$. The chemical structure of this indole alkaloid appeared to be relate to the structure of yohimbine and voacangine ${ }^{[2]}$.

\section{Pharmacology of Mitragynine and 7-Hydroxymitragynine}

While Ketum has many valuable therapeutic claims since the olden days, mitragynine has been identified to be involved and responsible for the unique properties of this medicinal plant. The effects and modes of actions of the alkaloid are very much morphine-like. However, as mitragynine differs structurally from morphine, the spectrum of pharmacological properties of mitragynine also differ ${ }^{[7]}$. The Methoxy- $(\mathrm{MeO})$ functional group which is present at the ninth carbon of the mitragynine structure is responsible for its anti-nociceptive proper-

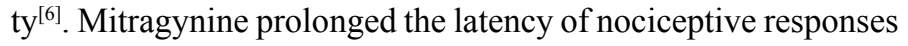
in hot plate, tail pinch test and inhibition of writhing respons$\mathrm{es}^{[7,11,12]}$. However, the minor alkaloid, 7-hydroxymitragynine also exhibited anti-nociceptive activities and showed 13 and 46 folds higher activity than morphine and mitragynine respectively ${ }^{[7,13]}$. The analgesic effects of both mitragynine and 7-hydroxy mitragynine primarily involve the activation of the opioid receptors. The binding assay studies have shown that mitragynine has a relatively highest affinity for $\mu$-opioid receptors followed by $\kappa$ - and $\delta$ - opioid receptors ${ }^{[1,15]}$ and in comparison to morphine, mitragynine has a higher affinity towards $\delta$ - and $\kappa$ - opioid receptors $^{[2]}$. A detailed study on the involvement of specific opioid receptor subtype showed unlike morphine, mitragynine exerted its anti-nociceptive property through both $\mu$ - and $\delta$ - opioid receptors ${ }^{[16]}$. The mitragynine inhibited the contraction of electrically stimulated guinea pig ileum ${ }^{[17]}$ and mouse vas deferens ${ }^{[18]}$ through opioid receptors in a dose-dependent manner. Despite these evidences for the interaction between mitragynine and the opioid receptors, mitragynine is also known to exert its physiological effects through various systems. The central monoaminergic system was shown to be involved in the pain modulation activity of mitragynine. The noxious stimulus test showed the descending noradrenergic and serotonergic systems modulates the anti-nociceptive activity of mitragynine on tail flick test (mechanical stimulation) while the descending noradrenergic system regulates the anti-nociceptive activity of mitragynine hot-plate test (thermal stimulation) ${ }^{[19]}$. The mitragynine suppression of head twitch response caused by 5-methoxy-N,N-dimethyltryptamine demonstrated the effect of mitragynine on serotonergic function in the brain ${ }^{[20]}$. Meanwhile, 7-hydroxymitragynine also exerted its effects through the opioid receptors, mainly the $\mu$ receptors ${ }^{[13,21]}$. In a tail-flick test, 7-hydroxymitragynine induced anti-nociceptive effects through the $\mu$ - receptors in mice ${ }^{[22]}$. The administration of 7-hydroxymitragynine also led to full substitution to the morphine's discriminate stimulus. This effect can be reversed by treatment with naloxone which demonstrated the involvement of opioid receptors ${ }^{[23]}$.

\section{The Dose-Dependent Effects of Mitragynine}

The effects of mitragynine changes depending upon the level of exposure, i.e. it is dose-dependent. The dual effects of mitragynine had been demonstrated in both rodent and human studies where at lower doses, it gave coca-like ecstatic effects while at higher doses it gave opioid-like depressant effects ${ }^{[3,23]}$. The administration of mitragynine also showed dose-dependent anti-nociceptive response in acetic acid-induced writhing test and formalin test ${ }^{[24]}$. The mitragynine inhibited the contraction of electrically stimulated guinea pig ileum ${ }^{[17]}$ and mouse vas deferens ${ }^{[18]}$ through opioid receptors in a dose-dependent manner. The mitragynine administration also showed significant dose-dependent increase in latency time in the hot plate test in mice ${ }^{[25,26]}$. Besides pain relieving property, the mitragynine has been found to have other various physiological effects in dose-dependent manner both in-vitro and in-vivo studies. A dose-dependent manner of inhibition of oxygenase followed by suppression of inflammatory mediator in RAW264.7 macrophage cells suggested the possible anti-inflammatory properties of mitragynine ${ }^{[27]}$. In a rodents study using anaesthetised rats, the dose-dependent inhibitory effects of mitragynine on 2-deoxy-D-glucose-stimulated gastric acid secretion also being confirmed ${ }^{[28]}$. Limited studies also demonstrated the effect of mitragynine in cognitive function. In mice, 28 days of chronic administration of mitragynine $(5,10$ and $15 \mathrm{mg} / \mathrm{kg})$ significantly impaired the working memory and reduced movement activity in open field test ${ }^{[4]}$. Mitragynine had a significant dose-dependent effect on locomotor activity where at low dose it resulted in hyperlocomotion and at high dose, hypolocomotion ${ }^{[3]}$. The same study observed dose-dependent anxiolytic effects and Conditioned Place Preference (CPP) which reflected the rewarding properties of mitragynine in mice $^{[3]}$.

An increase in the horizontal and vertical exploratory activity in the Y-maze was observed following acute oral exposure of mitragynine $(20,40$ and $80 \mathrm{mg} / \mathrm{kg}$ ) in Swiss albino mice $^{[29]}$. A recent study on the discriminative stimulus properties of mitragynine in rats showed that the mitragynine dose dependently substituted fully to the morphine discriminative 
stimulus ${ }^{[23]}$. Although this plant and its derivatives are known for their unique properties for posing both opioid- and psychostimulant-like drug effects, the exact central system and pathways which are involved in modulating the addictive reward process of mitragynine remains elusive.

\section{The Endocannabinoid System}

The Endocannabinoid (ECB) is a signalling system which consists of ECB ligands, receptors and enzymes which are responsible for their synthesis, hydrolysis and reuptake mechanism. The ligands of ECB system are anandamide, 2-arachidonic and 2-arachidonyl glycerol ${ }^{[30,31]}$. There are two subtypes of CBR, CB1R and CB2R. Although distributed throughout the body, the $\mathrm{CB} 1 \mathrm{R}$ can be found more predominantly in the brain while the $\mathrm{CB} 2 \mathrm{R}$ is more prominently found in the immune cells ${ }^{[32,33]}$. The CB1R is seven trans membrane domain G-protein coupled receptor, thus the G protein activation mediates the signal transduction. Although the meso corticolimbic dopamine system is globally known for its pivotal role in reinforcing effects of drugs of abuse, the ECB system also plays a significant part in drug-seeking reinforced by drug-related cues and drug-relapse. The CB1R is well known for its role in mediating the effects of the psychoactive compounds ${ }^{[32]}$. In CBR1 knock-out mice, studies had the demonstrated the mice failure to acquire self-administration and CPP, as well as less withdrawal syndrome to morphine, nicotine, cocaine, alcohol, psycho stimulants and $\Delta-9$-Tetrahydrocannabinoid $(\mathrm{THC})^{[34,35]}$. Thus, given the similar properties between mitragynine and morphine and the known crosstalk between opioids and ECBs, it is plausible to postulate that the cannabinoid receptors may also be involved in mitragynine drug-seeking and reinforcement motivational behaviour within the brain reward pathways.

\section{The Functional Interaction of Endogenous Cannabinoid and Opioid Systems}

Opiates and cannabis are two naturally occurring psychotropic drugs that are in use in human culture since the ancient times. Morphine which is being used as major analgesic, interacts with the ubiquitously distributed seven transmembrane G-protein coupled opioid receptors ${ }^{[36]}$. On the other hand, the psychoactive effects of THC are centrally mediated by activation of the cannabinoid receptor of the ECB system. In addition to opioid system, the ECB system has been shown to involve in exerting the anti-nociceptive features of morphine and vice versa. Besides the anti-nociceptive effects, the reciprocal interaction between the opioid-ECB system also exists in reinforcement effects ${ }^{[37-39]}$. Although the mitragynine is claimed to be opioid-like, however, the recent study on the involvement of ECB system in the anti-nociceptive property of acute mitragynine administration showed no participation of cannabinoid receptors $^{[26]}$. Nevertheless, the participation of the ECB in reinforcement and addictive effects of mitragynine is unknown.

\section{Cerebellum Functions: Beyond Motor Control}

Over several decades cerebellum is recognised as the maestro of motor control. The manifestation of motor in coordination of voluntary movement (termed ataxia) is linked to cerebellar lesions ${ }^{[40]}$. However, the notions on involvement of cerebellum in cognitive functions started to revolve and were being proposed by researchers two decades ago, particularly based from the findings of neuroimaging studies. The Positron Emission Topography (PET) study demonstrated the activation of cerebellum in relation to ejaculation ${ }^{[41]}$. Further detailed study on this showed ejaculation related stimulation of the cerebellar dentate nucleus and anterior vermis ${ }^{[42]}$. In a different study, the observation of an increased mid-vermis granule neurons activation in relation to sexual provocation and ejaculation in rats hinted the involvement of cerebellum in heightened emotional responses ${ }^{[43]}$. Besides cerebellum involvement in semantic discrimination and sexual behaviour, its role in learning and memory has also been implicated. A human PET imaging study conducted to correlate the brain region activation in relation to spatial memory and object recognition revealed activation of the cerebellum with regards to long term memory retrieval ${ }^{[44]}$. The cerebellum has been implicated in fear memories. Furthermore, the role of cerebellum for perceptual events has been studied where fMRI imaging of the brain during a temporal-spatial task showed the involvement of lobule VII Crus I which implied cerebellar involvement in perceptual predictions ${ }^{[45]}$.

\section{Implicating Cerebellum in Addiction}

In addition to executive, non-motor functions, evidences are accumulating for the role of cerebellum in development of drug addictive behaviour ${ }^{[40]}$. The basis to examine cerebellum and its role in cognitive functions and drug dependence can be tackled from the structural and functional connections the cerebellum has with various brain regions, such as the memory and brain rewards circuitry. The recorded neuronal responses in the amygdala and hippocampus following electrical stimulation of the cerebellar fastigial nucleus with relatively short delay times showed direct connections between the areas and cerebellum ${ }^{[46]}$. Furthermore, no delay was observed in the cerebellar vermis response time in fastigial nucleus in the lesioned cat ${ }^{[46]}$. In addition, human studies using the Transcranial Magnetic Stimulation (TMS) with stimulation across cerebellar vermis has resulted in an increased theta activity in the Pre-Frontal Cortex $(\mathrm{PFC})^{[47]}$. Findings of such studies further supported the earlier described cerebellar modulation of various cognitive, non-motor functions through functional pathways between cerebellum and other brain structures. Regarding the participation of cerebellum in addiction and relapse, emerging body of evidences suggested the fronto-cerebellar circuitry (Figure 1.1) to implicate cerebellum in the drug-seeking motivated behaviour. The PFC is wellknown for its involvement in executive controls such as decision making and emotion. The PFC is highly associated to the brain's reward circuit, the limbic system. The cerebellum, on the other hand, is interconnected to the PFC through the reciprocal connection between Brodmann area 9 and 46 of PFC and cerebellum via dentate nuclei which project through thalamus while the PFC sends feedback via the pontine nuclei ${ }^{[48]}$. The Brodmann area 9 which is also known as the dorsal PFC acts as one of the important component in regulating the reward process. 


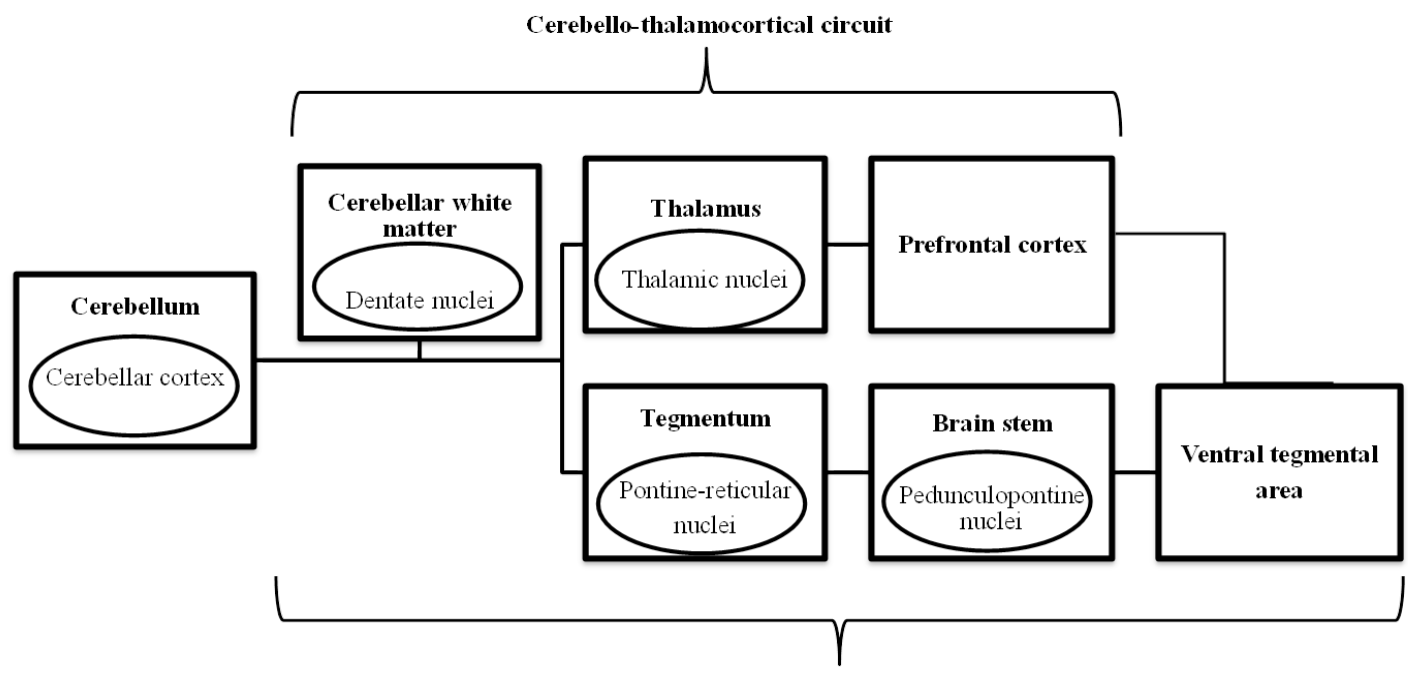

Cerebello-ventral tegmental circuit

Figure 1: The schematic illustration of the cerebello-thalamocortical and cerebello-ventral tegmental circuit pathway. In both circuits, the projections originate from the cerebellar cortex which projects into the dentate nuclei of cerebellar white matter. In the cerebello-thalamocortical circuit, the projection from dentate nuclei will projects to the thalamic nuclei of thalamus and terminates in the prefrontal cortex. In cerebello-ventral tegmental circuit, the projection from dentate nuclei will project into the pontine-reticular nuclei of tegmentum that in turn projects into ventral tegmental area via pedunculopontine of brain stem. The VTA dopaminergic cell bodies finally terminate in the PFC.

Moreover, the cerebellar projections also projects to the mesolimbic Ventral Tegmental Area (VTA) via cerebellar dentate nuclei which pass thorough the reticulo-tegmental nuclei that project to pedunculopontine nuclei. The VTA dopaminergic cell bodies finally terminate in the PFC (Figure 1.1) ${ }^{[49-52]}$. Taken together, these anatomical connections suggest the reciprocal interaction between the PFC and the cerebellum in cognition regulation as well as the possible dopamine release in mesolimbic VTA and in PFC activated by the cerebellar projection.

The relevance of the PFC and cerebellum working in parallel has been shown through data from several studies. The alterations or diminished fronto-cerebellar circuitry in relation to drug usage has been observed. The fMRI study on working memory of alcoholics showed greater activation of the frontal together with the activation of cerebellar regions in order to achieve the equal performance level as compared with controls ${ }^{[53]}$. Similarly, the study on the working memory of cocaine users showed cerebellar hyperactivity to compensate the hypoactivity of cingulate cortex ${ }^{[54]}$. Analysis of alcohol dependents showed activation of dorsolateral PFC-cerebellar VIII system during resting state while the dorsolateral PFC-cerebellar VI system were activated during task engaged. The observed results were in parallel to normal subjects and thus added evidence for the recruitment of cerebellar-based functional networks as a compensatory act by the brain for normal functionality ${ }^{[55]}$. The study on subordinate hand finger tapping task in alcohol abstainers correlated with the deficits in functionality of pre motor Brodmann area 6 and cerebellar Lobule VI as well as prefrontal Brodmann area 9 and cerebellar Lobule VIII ${ }^{[56]}$. The fMRI study on the neural activity and functional reorganisation in heroin dependent subjects showed decreased amplitude of low frequency fluctuation in prefrontal regions accompanied by increase in cerebellar region ${ }^{[57]}$. Also, the cerebellum encodes an internal model that reproduces the properties of PFC. Therefore, in the event of decrease in the functionality of PFC the cerebellum which has previously stored the information from PFC will be activated to represent the $\mathrm{PFC}^{[58]}$. Collectively, these findings imply cerebellar activation in the event of reduced prefrontal control activity. These anatomico-functional arguments lend cohesive arguments in extending the involvement of cerebellum in reward paradigm and modulation of drug-seeking behaviour.

The plausible leads for the involvement of cerebellum in drug seeking behaviour can be seen from the dopaminergic neurons in the cerebellum. The observation of a decreased in the level of cerebellar dopamine following lesions in the midbrain dopaminergic regions, proved that the dopaminergic cell bodies projected from the retrorubal-substania nigra-VTA to the cerebellum $^{[59]}$. The presence of dopaminergic A10 group in both primates $^{[60]}$ and rodents ${ }^{[61]}$ has been identified. The projection of A10 cell group from VTA into rat cerebellar cortex terminates at the GL and PL ${ }^{[61]}$. In an immune cytochemical study, the widespread innervations of dopamine fibres in the rat cerebellum reported presence of intense amount of dopamine afferent fibres in the $\mathrm{ML}^{[62]}$. The study also demonstrated the presence of dopamine membrane transporters in the cerebellar vermis, granule cell layers ${ }^{[63]}$. The study on the human cerebellum revealed the presence of mRNA for Dopamine Transporter (DAT) in the structure $^{[64]}$. While the cerebellum receives projections from the VTA, the VTA itself receives cathecolamine fibres ${ }^{[65]}$ and fibre from lateral and interpositus cerebellar nuclei ${ }^{[61]}$.

Besides these, analogous to the limbic circuit dopamine, the cerebellar dopamine system has also been observed to participate in drug related activities. Klitenick and co-workers showed increased c-fos expression in rat cerebellum following administration of cocaine and dextro amphetamine. The expression was eradicated by SCH233390, D1 receptor antagonist ${ }^{[66]}$. In addition, an upregulation of cerebellar D2-like receptors was detected following inoculation of dextro amphetamine in rats ${ }^{[67]}$. Cocaine has been reported to sensitise c-fos mRNA in the cerebellum with the aid of dopamine D1 and D2 receptors ${ }^{[68]}$. Together, these results suggest that the cerebellum is likely to be involved in development of addictive behaviour. 
Moreover, in relation to the endocannabinoid system, the CB1R that are widely spread in brain with a vast expression in the cerebellum ${ }^{[69,70]}$. The $\mathrm{CB} 1 \mathrm{R}$ are highly expressed in ML, Climbing Fibre (CF), Parallel Fibre (PF) and BC especially at the $\mathrm{BC}-\mathrm{PC}$ synapse $\mathrm{e}^{[71]}$. Meanwhile, in younger animals, the presence of $\mathrm{CB} 1 \mathrm{R}$ has been detected in axons and synaptic terminals of cerebellar $\mathrm{BC}, \mathrm{SC}$ and $\mathrm{PF}$ and $\mathrm{CF}^{[72]}$. The $\mathrm{CB} 1 \mathrm{R}$ are expressed predominantly at presynaptic of the neurons that synapse onto PC. The Long Term Depression (LDP) and Long Term Potentiation (LTP) represent the key candidate mechanisms for learning and memory processes associated with addiction ${ }^{[73,74]}$. The cerebellar CB1R in PF regulates short term plasticity and $\mathrm{LTD}^{[75]}$. Thus, the CB1R induced cerebellar synaptic plasticity may causatively contribute to drug addictive behaviour.

The role of CB1 in modulating the dopaminergic system is known as one of the mechanisms in drug addiction. The inhibition of Gamma-Aminobutyric Acid (GABA) release will cause an increase in the dopamine release $\mathrm{e}^{[76-78]}$. In cerebellum, the $\mathrm{CB} 1 \mathrm{R}$ are localised in presynaptic GABAergic neurons. The signalling of these presynaptically expressed $\mathrm{CB} 1 \mathrm{R}$ could depress the GABA release which in turn increases the dopamine level. Thus, within the cerebellum, the activation of presynaptically located CB1R possibly depresses GABA release and eventually disinhibits the dopaminergic system. This shows a predominant role of cerebellar CB1R in inactivating the GABAergic pathways and contributes to the development of addictive behaviour.

The role of ECB system as retrograde messenger candidates in cerebellum has been demonstrated. In a study, the activation of CF of Naval Medical Research Institute (NMRI) mice brain slices triggers the production of ECBs. The post synaptically produced ECBs bind to presynaptic CB1R which in turn inhibit the GABAergic input to PC. The reversal of this effect by CB1R antagonist, rimonabant further validates the role of CB1R as retrograde messenger in cerebellum ${ }^{[79]}$. Overall, the CB1R mediated cerebellar plasticity and fine-tuned regulation of neural transmission may also contribute to the development of drug addiction, dependence and relapse.

\section{Conclusion}

The emerging new psychoactive substances, in particular the phytochemical of plant mitragynine from Mitragyna speciosa (Ketum) has become a global concern. Mitragynine's potential as a psychoactive plant appeared anecdotally as an opium substitute and has since emerged with an abuse liability. Targets within the brain-reward circuitry are implicated in drug-induced synaptic plasticity of addiction. The involvement of ECB system in positive modulatory effects triggering the addiction and relapse has been assessed. Also, the ECB system represents a novel class of signalling molecules that contribute to short- and long-term synaptic plasticity throughout the brain through the interaction of CB1R. The interaction between opioids and ECB system in reinforcing effects of addictive drugs and in modulating the synaptic plasticity has been elaborated.

Although the meso corticolimbic dopaminergic region has always been recognised to be central to reward processing, recent evidences implicate the involvement of the cerebellum in cognitive functions with an emerging role in the transition from a pattern of recreational drug taking to the development of an addictive pattern of behaviour. The neuroplasticity changes in the cerebellum have been shown to be mediated by endocannabinoid-dependent cellular mechanisms. The ubiquitous CB1R in cerebellum could be the main signalling molecule that may mediate the neuroplasticity changes in the cerebellum contributing for the involvement of the cerebellum in drug-induced conditioned memory. Thus, given mitragynine's opioid agonism, it is postulated that the CB1R within the cerebellum may well represent the novel neurochemical basis of mitragynine/Ketum abuse liabilities. Further research is warranted to ascertain the role of CB1R as potential candidate within the cerebellum and its connection with the brain rewards circuitry.

\section{References}

1. Gong, F., H-p, Gu., Q-t, Xu., et al. Genus Mitragyna: Ethnomedicinal uses and pharmacological studies. (2012) Phytopharmacology 3(2): 263-272.

2. Hassan, Z., Muzaimi, M., Navaratnam, V., et al. From Kratom to mitragynine and its derivatives: physiological and behavioural effects related to use, abuse, and addiction. (2013) Neurosci Biobehav Rev 37(2): 138-151.

3. Yusoff, N.H., Suhaimi, F.W., Vadivelu, R.K., et al. Abuse potential and adverse cognitive effects of mitragynine (kratom). (2014) Addiction biology.

4. Chittrakarn, S., Sawangjaroen, K., Prasettho, S., et al. Inhibitory effects of kratom leaf extract (Mitragyna speciosa Korth.) on the rat gastrointestinal tract. (2008) J Ethnopharmacol 116(1): 173-178.

5. Apryani, E., Hidayat, M.T., Moklas, M.A., et al. Effects of mitragynine from Mitragyna speciosa Korth leaves on working memory. (2010) J Ethnopharmacol. 129(3): 357-360.

6. Stolt, A.C., Schroder, H., Neurath, H., et al. Behavioral and neurochemical characterization of kratom (Mitragyna speciosa) extract. (2014) Psychopharmacology 231(1): 13-25.

7. Adkins, J.E., Boyer, E.W., McCurdy, C.R. Mitragyna speciosa, a psychoactive tree from Southeast Asia with opioid activity. (2011) Curr Top Med chem 11(9): 1165-1175.

8. Takayama, H. Chemistry and pharmacology of analgesic indole alkaloids from the rubiaceous plant, Mitragyna speciosa. (2004) Chem Pharm Bull 52(8): 916-928.

9. Babu, K.M., McCurdy, C.R., Boyer, E.W. Opioid receptors and legal highs: Salvia divinorum and Kratom. (2008) Clin Toxicol (Phila) 46(2): 146-152.

10. Zacharias, D., Rosenstein, R., Jeffrey, G. The structure of mitragynine hydroiodide. (1965) Acta Crystallographica 18(6): 1039-1043.

11. Liu, H., McCurdy, C.R., Doerksen, R.J. Computational Study on the Conformations of Mitragynine and Mitragynaline. (2010) Theochem 945(1-3): 57-63.

12. Reanmongkol, W., Keawpradub, N., Sawangjaroen, K. Effects of the extracts from Mitragyna speciosa Korth. leaves on analgesic and behavioral activities in experimental animals. (2007) Songklanakarin J Sci Technol 29(Suppl 1): 39-48.

13. Sabetghadam, A., Ramanathan, S., Mansor, S.M. The evaluation of antinociceptive activity of alkaloid, methanolic, and aqueous extracts of Malaysian Mitragyna speciosa Korth leaves in rats. (2010) Pharmacognosy Res 2(3): 181-185.

14. Matsumoto, K., Horie, S., Ishikawa, H., et al. Antinociceptive effect of 7-hydroxymitragynine in mice: Discovery of an orally active opioid analgesic from the Thai medicinal herb Mitragyna speciosa. (2004) Life sciences 74(17): 2143-2155.

15. Yamamoto, L.T., Horie, S., Takayama, H., et al. Opioid receptor agonistic characteristics of mitragynine pseudoindoxyl in comparison with mitragynine derived from Thai medicinal plant Mitragyna speciosa. (1999) Gen Pharmacol 33(1): 73-81.

16. Boyer, E.W., Babu, K.M., Adkins, J.E. Self-treatment of opioid withdrawal using kratom (Mitragynia speciosa korth). (2008) Addiction 
103(6): 1048-1050.

17. Thongpradichote, S., Matsumoto, K., Tohda, M., et al. Identification of opioid receptor subtypes in antinociceptive actions of supraspinally-administered mitragynine in mice. (1998) Life sciences 62(16): 1371-1378.

18. Watanabe, K., Yano, S., Horie, S. Inhibitory effect of mitragynine, an alkaloid with analgesic effect from Thai medicinal plant Mitragyna speciosa, on electrically stimulated contraction of isolated guinea-pig ileum through the opioid receptor. (1997) Life sciences 60(12): 933942.

19. Matsumoto, K., Yamamoto, L.T., Watanabe, K., et al. Inhibitory effect of mitragynine, an analgesic alkaloid from Thai herbal medicine, on neurogenic contraction of the vas deferens. (2005) Life sciences 78(2):187-194.

20. Matsumoto, K., Mizowaki, M., Suchitra, T., et al. Central antinociceptive effects of mitragynine in mice: contribution of descending noradrenergic and serotonergic systems. (1996) Eur J Pharmacol 317(1): 75-81.

21. Matsumoto, K., Mizowaki, M., Takayama, H., et al. Suppressive effect of mitragynine on the 5-methoxy-N, N-dimethyltryptamine-induced head-twitch response in mice. (1997) Pharmacol Biochem Behav 57(1): 319-323.

22. Matsumoto, K., Hatori, Y., Murayama, T., et al. Involvement of mu-opioid receptors in antinociception and inhibition of gastrointestinal transit induced by 7-hydroxymitragynine, isolated from Thai herbal medicine Mitragyna speciosa. (2006) Eur J pharmacol 549(1-3): 63-70. 23. Matsumoto, K., Horie, S., Takayama, H., et al. Antinociception, tolerance and withdrawal symptoms induced by 7-hydroxymitragynine, an alkaloid from the Thai medicinal herb Mitragyna speciosa. (2005) Life sciences 78(1): 2-7.

24. Harun, N., Hassan, Z., Navaratnam, V., et al. Discriminative stimulus properties of mitragynine (kratom) in rats. (2015) Psychopharmacology 232(13): 2227-2238.

25. Shaik Mossadeq, W.M., Sulaiman, M.R., Tengku Mohamad, TA., et al. Anti-inflammatory and antinociceptive effects of Mitragyna speciosa Korth methanolic extract. (2009) Med Princ Pract 18(5): 378-384.

26. Sabetghadam, A., Navaratnam, V., Mansor, S.M. Dose-Response Relationship, Acute Toxicity, and Therapeutic Index between the Alkaloid Extract of Mitragyna speciosa and Its Main Active Compound Mitragynine in Mice. (2013) Drug Development Research 74(1): 23-30.

27. Shamima, A.R., Fakurazi, S., Hidayat, M.T., et al. Antinociceptive Action of Isolated Mitragynine from Mitragyna Speciosa through Activation of Opioid Receptor System. (2012) International Journal of Molecular Sciences 13(9): 11427-11442.

28. Utar, Z., Majid, M.I., Adenan, M.I., et al. Mitragynine inhibits the COX-2 mRNA expression and prostaglandin E(2) production induced by lipopolysaccharide in RAW264.7 macrophage cells. (2011) J Ethnopharmacol 136(1): 75-82.

29. Tsuchiya, S., Miyashita, S., Yamamoto, M., et al. Effect of mitragynine, derived from Thai folk medicine, on gastric acid secretion through opioid receptor in anesthetized rats. (2002) Eur J pharmacol 443(1): 185-188.

30. Hazim, A.I., Mustapha, M., Mansor, S.M. The effects on motor behaviour and short-term memory tasks in mice following an acute administration of Mitragyna speciosa alkaloid extract and mitragynine. (2011) Journal of Medicinal Plants Research 5(24): 5810-5817.

31. Hanus, L., Abu-Lafi, S., Fride, E., et al. 2-arachidonyl glyceryl ether, an endogenous agonist of the cannabinoid CB1 receptor. (2001) Proc Natl Acad Sci USA 98(7): 3662-3665.

32. Fride, E. Endocannabinoids in the central nervous system--an overview. (2002) Prostaglandins Leukot Essent Fatty Acids 66(2-3): 221233.

33. Kreitzer, AC., Regehr, WG. Retrograde signaling by endocannabinoids. (2002) Curr Opin Neurobiol 12(3): 324-330.

34. Maldonado, R., Valverde, O., Berrendero, F. Involvement of the endocannabinoid system in drug addiction. (2006) Trends Neurosci 29(4): 225-232.
35. Pacher, P., Batkai, S., Kunos, G. The endocannabinoid system as an emerging target of pharmacotherapy. (2006) Pharmacol Rev 58(3): $389-462$.

36. Justinova, Z., Panlilio, L.V., Goldberg, S.R. Drug addiction. (2009) Current topics in behavioral neurosciences. 1: 309-346.

37. Waldhoer, M., Bartlett, S.E., Whistler, J.L. Opioid receptors. (2004) Annu Rev Biochem 73: 953-990.

38. Ledent, C., Valverde, O., Cossu, G., et al. Unresponsiveness to cannabinoids and reduced addictive effects of opiates in $\mathrm{CB} 1$ receptor knockout mice. (1999) Science 283(5400): 401-404.

39. Solinas, M., Goldberg, S.R. Involvement of mu-, delta- and kappa-opioid receptor subtypes in the discriminative-stimulus effects of delta-9-tetrahydrocannabinol (THC) in rats. (2005) Psychopharmacology 179(4): 804-812.

40. Solinas, M., Goldberg, S.R. Motivational effects of cannabinoids and opioids on food reinforcement depend on simultaneous activation of cannabinoid and opioid systems. (2005) Neuropsychopharmacology 30(11): 2035-2045.

41. Miquel, M., Toledo, R., Garcia, L.I., et al. Why should we keep the cerebellum in mind when thinking about addiction? (2009) Current Drug Abuse Rev 2(1): 26-40.

42. Holstege, G., Georgiadis, J.R., Paans, A.M., et al. Brain activation during human male ejaculation. (2003) J Neurosci 23(27): 9185-9193. 43. Georgiadis, J.R., Reinders, A.A., Van der Graaf, F.H., et al. Brain activation during human male ejaculation revisited. (2007) Neuroreport 18(6): 553-557.

44. Manzo, J., Miquel, M., Toledo, R., et al. Fos expression at the cerebellum following non-contact arousal and mating behavior in male rats. (2008) Physiol Behav 93(1-2): 357-363.

45. Moscovitch, C., Kapur, S., Kohler, S., et al. Distinct neural correlates of visual long-term memory for spatial location and object identity: a positron emission tomography study in humans. (1995) Proc Natl Acad Sci USA 92(9): 3721-3725.

46. O'Reilly, J.X., Mesulam, M.M., Nobre, A.C. The cerebellum predicts the timing of perceptual events. (2008) J Neurosci 28(9): 22522260 .

47. Heath, R.G., Dempesy, CW., Fontana, C.J., et al. Cerebellar stimulation: effects on septal region, hippocampus, and amygdala of cats and rats. (1978) Biol Psychiatry 13(5): 501-529.

48. Schutter, D.J., Van Honk, J. An electrophysiological link between the cerebellum, cognition and emotion: frontal theta EEG activity to single-pulse cerebellar TMS. (2006) NeuroImage 33(4): 1227-1231.

49. Middleton, F.A., Strick, P.L. Cerebellar projections to the prefrontal cortex of the primate. (2001) J Neurosci 21(2): 700-712.

50. Snider, R.S., Maiti, A., Snider, S.R. Cerebellar pathways to ventral midbrain and nigra. (1976) Exp Neurol 53(3): 714-728.

51. Fallon, J.H., Moore, R.Y. Catecholamine innervation of the basal forebrain. IV. Topography of the dopamine projection to the basal forebrain and neostriatum. (1978) J Comp Neurol 180(3): 545-580.

52. Perciavalle, V., Berretta, S., Raffaele, R. Projections from the intracerebellar nuclei to the ventral midbrain tegmentum in the rat. (1989) Neuroscience 29(1): 109-119.

53. Rogers, T.D., Dickson, P.E., Heck, D.H. et al. Connecting the dots of the cerebro-cerebellar role in cognitive function: neuronal pathways for cerebellar modulation of dopamine release in the prefrontal cortex. (2011) Synapse 65(11): 1204-1212.

54. Desmond, J.E., Chen, S.H.A., DeRosa, E., et al. Increased frontocerebellar activation in alcoholics during verbal working memory: an fMRI study. (2003) NeuroImage 19(4): 1510-1520.

55. Hester, R., Garavan, H. Executive dysfunction in cocaine addiction: evidence for discordant frontal, cingulate, and cerebellar activity. (2004) J Neurosci 24(49): 11017-11022.

56. Chanraud, S., Pitel, A-L., Müller-Oehring, E.M., et al. Remapping the Brain to Compensate for Impairment in Recovering Alcoholics. (2013) Cereb Cortex 23(1): 97-104.

57. Rogers, B.P., Parks, M.H., Nickel, M.K., et al. Reduced fronto-cerebellar functional connectivity in chronic alcoholic patients. (2012) Al- 
cohol Clin Exp Res 36(2): 294-301.

58. Wang, Y., Zhu, J., Li, Q., et al. Altered fronto-striatal and fronto-cerebellar circuits in heroin-dependent individuals: a resting-state FMRI study. (2013) PLoS One 8(3):e58098.

59. Ito, M. Cerebellar circuitry as a neuronal machine. (2006) Prog Neurobiol 78(3-5): 272-303.

60. Kizer, J.S., Palkovits, M., Brownstein, M.J. The projections of the A8, A9 and A10 dopaminergic cell bodies: evidence for a nigral-hypothalamic-median eminence dopaminergic pathway. (1976) Brain Res 108(2): 363-370.

61. Felten, D.L., Sladek, JR.Jr. Monoamine distribution in primate brain V. Monoaminergic nuclei: anatomy, pathways and local organization. (1983) Brain Res Bull 10(2): 171-284.

62. Ikai, Y., Takada, M., Shinonaga, Y., et al. Dopaminergic and non-dopaminergic neurons in the ventral tegmental area of the rat project, respectively, to the cerebellar cortex and deep cerebellar nuclei. (1992) Neuroscience 51(3): 719-728.

63. Panagopoulos, N.T., Papadopoulos, G.C., Matsokis, N.A. Dopaminergic innervation and binding in the rat cerebellum. (1991) Neurosci Lett 130(2): 208-212.

64. Melchitzky, D.S., Lewis, D.A. Tyrosine Hydroxylase- and Dopamine Transporter-Immunoreactive Axons in the Primate Cerebellum: Evidence for a Lobular- and Laminar-Specific Dopamine Innervation. (2000) Neuropsychopharmacology 22(5): 466-472.

65. Giompres, P., Delis, F. Dopamine transporters in the cerebellum of mutant mice. (2005) Cerebellum 4(2): 105-11.

66. Snider, RS., Maiti, A. Cerebellar contributions to the Papez circuit. (1976) J Neurosci Res 2(2): 133-146.

67. Klitenick, M.A., Tham, C.S., Fibiger, H.C. Cocaine and d-amphetamine increase c-fos expression in the rat cerebellum. (1995) Synapse 19(1): 29-36.

68. Bortolozzi, A., Duffard, R., Antonelli, M., et al. Increased sensitivity in dopamine $\mathrm{D}$ (2)-like brain receptors from 2,4-dichlorophenoxyacetic acid (2,4-D)-exposed and amphetamine-challenged rats. (2002) Ann N Acad Sci 965: 314-323.
69. Couceyro, P., Pollock, K.M., Drews, K., et al. Cocaine differentially regulates activator protein-1 mRNA levels and DNA-binding complexes in the rat striatum and cerebellum. (1994) Mol Pharmacol 46(4): 667-676.

70. Tsou, K., Brown, S., Sanudo-Pena, M.C,. et al. Immunohistochemical distribution of cannabinoid $\mathrm{CB} 1$ receptors in the rat central nervous system. (1998) Neuroscience 83(2): 393-411.

71. Kawamura, Y., Fukaya, M., Maejima, T., et al. The CB1 cannabinoid receptor is the major cannabinoid receptor at excitatory presynaptic sites in the hippocampus and cerebellum. (2006) J Neurosci 26(11): 2991-3001.

72. Mackie K. Distribution of cannabinoid receptors in the central and peripheral nervous system. (2005) Handb Exp Pharmacol (168): 299325.

73. Diana, M.A., Levenes, C., Mackie, K., et al. Short-term retrograde inhibition of GABAergic synaptic currents in rat Purkinje cells is mediated by endogenous cannabinoids. (2002) J Neurosci 22(1): 200-208.

74. Kauer, J.A., Malenka, R.C. Synaptic plasticity and addiction. (2007) Nat Rev Neurosci 8(11): 844-858.

75. Marsicano, G., Lafenêtre, P. Roles of the Endocannabinoid System in Learning and Memory. (2009) Curr Top Behav Neurosci 1: 201-230. 76. Carey, M.R., Myoga, M.H., McDaniels, K.R., et al. Presynaptic CB1 receptors regulate synaptic plasticity at cerebellar parallel fiber synapses. (2011) J Neurophysiol 105(2): 958-963.

77. Kalivas, P.W., Duffy, P., Eberhardt, H. Modulation of A10 dopamine neurons by gamma-aminobutyric acid agonists. (1990) J Pharmacol Exp Ther 253(2): 858-866.

78. Johnson, S.W., North, R.A. Opioids excite dopamine neurons by hyperpolarization of local interneurons. (1992) J Neurosci 12(2): 483488.

79. Bonci, A., Malenka, R.C. Properties and plasticity of excitatory synapses on dopaminergic and GABAergic cells in the ventral tegmental area. (1999) J Neurosci 19(10): 3723-3730. 